\title{
Drying Kinetics of Particulate Corundum Layers
}

\author{
K. CMiel ${ }^{a, *}$, J.J. MilczAreK ${ }^{a}$, L.C. BAM ${ }^{b}$, I.M. FiJAe-KIREJCZYK ${ }^{a}$, Z. Jurkowski ${ }^{a}$ \\ AND J. ŻOŁĄDEK ${ }^{a}$ \\ ${ }^{a}$ National Centre for Nuclear Research, A. Sołtana 7, 05-400 Otwock-Świerk, Poland \\ ${ }^{b}$ Radiation Science, Necsa, Church Street West ext., Pelindaba, Pretoria, 0001, South Africa
}

\begin{abstract}
The results of studies on drying of layers of particulate corundum performed with digital neutron radiography are presented. The statistical analysis of images was employed to reveal main features of the drying process. It is shown that the first period of drying proceeds within the whole body of the sample. Marked signatures of the end of first period of drying were found for sample mass, temperature, average image brightness as well as standard deviation of brightness time evolutions for the system composed of fine grains. The chaotic nature of this period is delineated by a distinct maximum in the standard deviation of sample image brightness. The presence of the drying front moving from the open surface to the sample bottom is proven to be an attribute of the advanced drying period.
\end{abstract}

DOI: 10.12693/APhysPolA.124.1029

PACS: 61.05.Tv, 81.05.Rm, 87.59.bf

\section{Introduction}

The drying process is typically divided into three main stages that are characterized by different rates of mass decrease of drying medium [1-4]. During the first period of drying (constant rate period - CRP), the rate of water loss is approximately constant and the spatial distribution of the water content declines uniformly within the entire volume of the sample. In the following two periods (falling rate periods - FRP) a gradual decrease of the rate of water loss is found and a discernible drying front moving from the open sample surfaces develops.

The aim of this work was to find main features of drying of wet particulate material by observations of water migration inside the volume of the sample. For comparison the experiments were performed for two particulate systems made of the same substance but composed of grains of two distinctly different sizes. The main method used was the thermal neutron imaging [5-10] which proved its usefulness in research on drying. The merit of the neutron radiography technique stems from the enormous scattering of thermal neutrons on hydrogen nuclei. In our studies the measurements of usual macroscopic parameters - mass and sample temperature were carried out simultaneously with neutron radiographs registration. The statistical analysis of sample images [6-9] was performed in order to describe quantitatively the emerging patterns of water distribution within sample and identify them within the common classification of the drying periods.

\section{Experiments}

The experiments were carried out at the neutron radiography station located at the $30 \mathrm{MW}$ nuclear research M RI of NCBJ [5-9].

*corresponding author; e-mail: katarzyna.cmiel@ncbj.gov.pl
The drying process was observed with on-line registration of the sample mass and temperature as well as sequences of neutron radiographs over the whole process. The samples were dried in the drying tunnel with $\approx 90^{\circ} \mathrm{C}$ hot air flowing at a rate of $\approx 2 \mathrm{~m} \mathrm{~s}^{-1}$. The sample was a rectangular block of $50 \mathrm{~mm} \times 50 \mathrm{~mm} \times 5.4 \mathrm{~mm}$ completely filled with a particulate material completely saturated with deionized water. Only the upper surface of the container was left open to the drying air. As the material the electro-corundum $\left(99 \% \mathrm{l}_{2} \mathrm{O}_{3}\right)$ of two different particle sizes was used. The coarse grain corundum (denoted as F20) consisted of grains of 1-1.11 mm diameter, whereas the size of grains forming the fine grain material (denoted as F220) was within 53-75 $\mu$ m interval.

Each sample was placed inside a vertical drying tunnel with its flat $50 \times 50 \mathrm{~mm}^{2}$ front surface at a distance of $\approx 70 \mathrm{~mm}$ position parallel to the converter screen (Fig. 1). For recording neutron images the high sensitivity CCD camera, Hamamatsu ORC -ER was used. The exposition time of $1.6 \mathrm{~s}$ was for any single frame. The projection ratio was 153 or $67 \mu \mathrm{m} /$ pixel. The image analysis was performed with the HiPic, SigmaScan Pro 5.0 .0 , and ImageJ $1.43 \mathrm{u}$ analytical software packages.

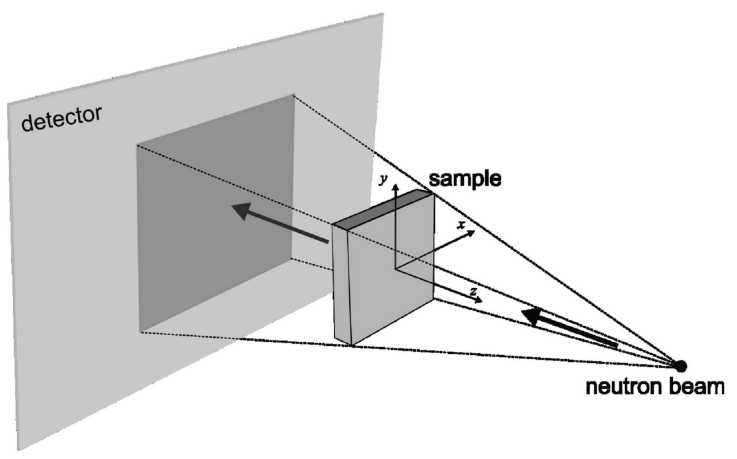

Fig. 1. The sample arrangement in the neutron radiography station. 
During experiments the sample mass, temperature inside the sample container and neutron radiographs were collected in the real time mode. The mass and temperature were recorded at 10 and $0.1 \mathrm{~s}$ intervals, respectively. The sequences of neutron radiographs were acquired at $5 \mathrm{~s}$ intervals during the first $2 \mathrm{~h}$ of the experiment and approximately every $300 \mathrm{~s}$ after that period.

\section{Experimental results}

The mass of investigated samples decreased with time for both types of material. The linear time dependence period could be satisfactorily distinguished only for fine grain sample whereas for coarse grain material the concave plot of mass vs. time was found (Fig. 2).

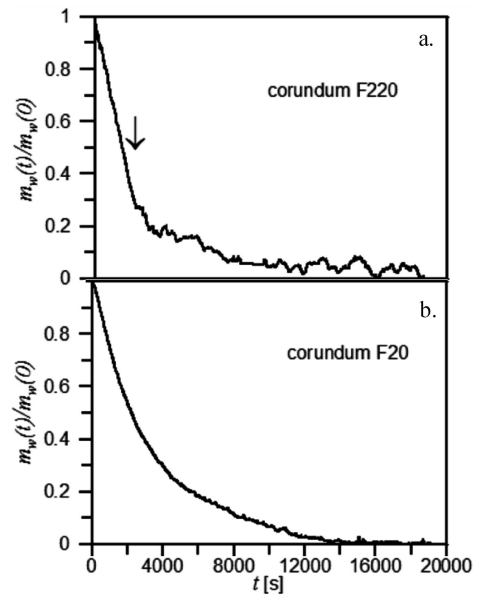

Fig. 2. Time dependence of the reduced mass content of water contained in the corundum sample of (a) F220 and (b) F20 grain size. The arrow in (a) indicates the end of the CRP. The water mass is divided by the mass of water contained in completely saturated sample.

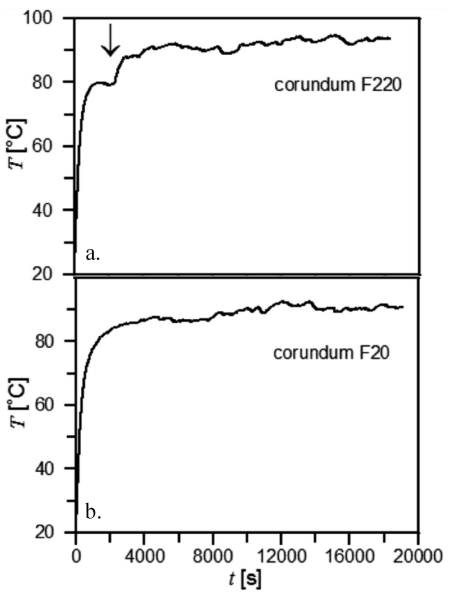

Fig. 3. Time dependence of the sample temperature during drying for (a) F220 and (b) F20 grain size. The arrow in (a) indicates the end of the CRP.

The time dependence of the temperature inside sample indicated quite clearly the presence of the first (CRP) period of drying with almost non-changing temperature only for the fine grain corundum (Fig. 3a). Even a small dip in the temperature vs. time dependence was observed at the end of the CRP at $\approx 2300 \mathrm{~s}$. No signs of such step-like plot of temperature vs. time was observed for the large grain size material (Fig. 3b).

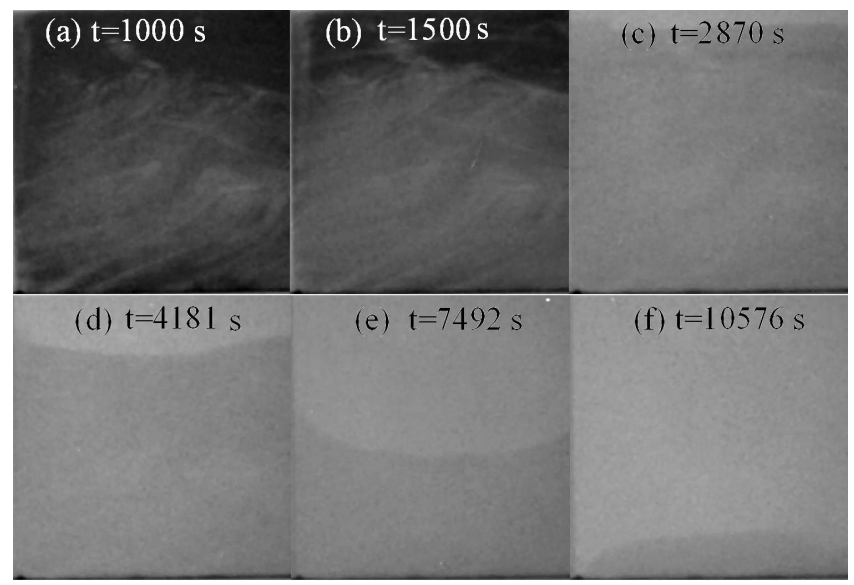

Fig. 4. The sample images during drying of the corundum F220 sample.
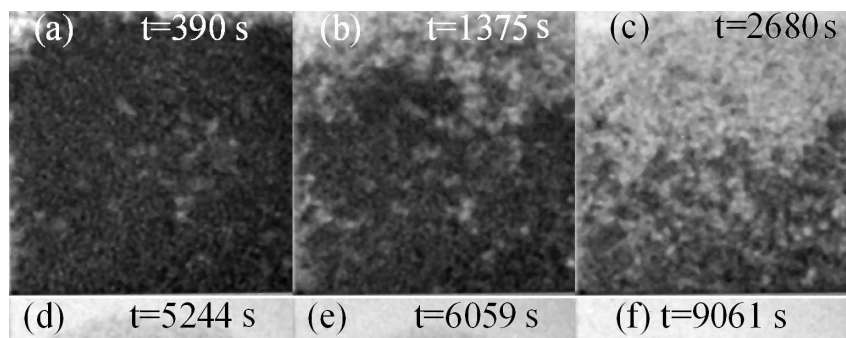

(e)

$t=6059 \mathrm{~s}$

(f) $\mathrm{t}=9061 \mathrm{~s}$

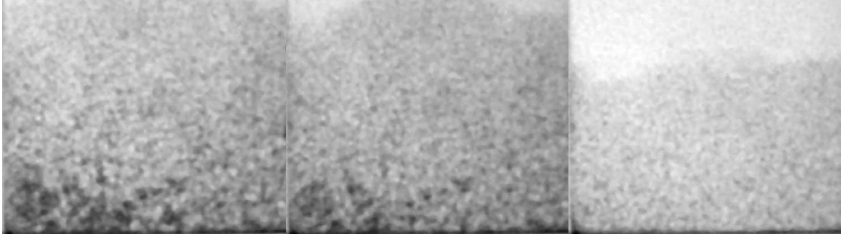

Fig. 5. The sample images during drying of the corundum F20 sample.

Before analysis the neutron radiographs were appropriately cropped to obtain the images of the whole sample only. Then the images were filtered to remove noise, and normalized including the black current and flat field corrections according to standard procedure [6-9]. The analysis of the sample pictures was carried out with HiPic and Image J software packages. The significant difference between the images corresponding to both types of particle size (Figs. 4 and 5) was found. For fine grained sample the image is much more uniform than the one obtained for the coarse grained one. The drying process proceeds in much more chaotic and turbulent way for the coarse grained sample. However, it should be noted that the drying seems to start from inside of the sample in each 


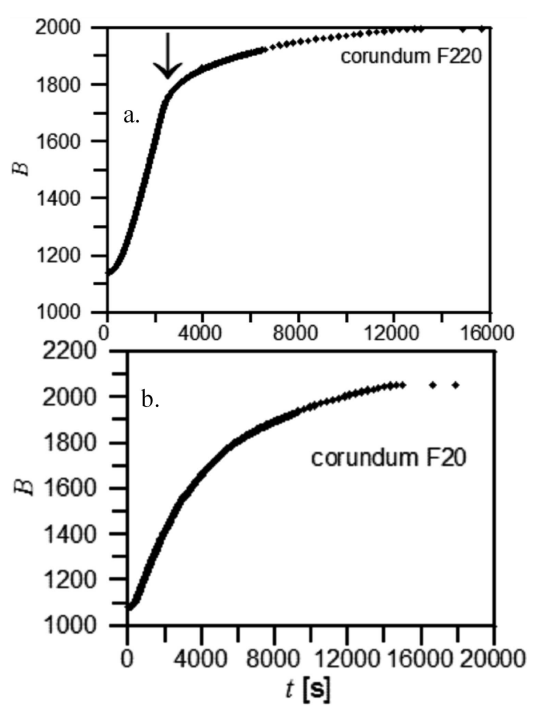

Fig. 6. Time dependence of the average brightness of the image of (a) F220 and (b) F20 grain size samples. The arrow in (a) indicates the end of the CRP.

case. Further on, the discernible drying front was found in the later periods of drying in both cases.

The average brightness of the sample images increases with the time of drying for each sample (Fig. 6). However, there is a marked difference between the smooth plot of the average brightness for the coarse grained sample (Fig. 6b) and the plot for the fine grained one which exhibits two regions of different slope (Fig. 6a).

\section{Analysis and discussion}

The statistical approach to the neutron radiographs was applied in order to quantify the features of the drying process [6-9]. In particular the variations of the standard deviation (STD) of the whole sample image brightness was analyzed. The presence of two maxima in plots of standard deviation vs. time was found for the fine grained medium (Fig. 7a) whereas only one maximum with extended wing for advanced times was found in coarse grained material (Fig. 7b). The first sharp maximum can be attributed to the increase of diversification of brightness due to intensive drying of inner regions of the sample. The increase in brightness in the middle of the sample image is conspicuous for both samples (Fig. 4a and $\mathrm{b}$ and Fig. 5a and $\mathrm{b}$ ). The second maximum in the brightness STD is a signature of the drying front travelling across the sample from the open top surface (Fig. 4c-e, Fig. 5c-e). In the case of the coarse grain sample the drying front develops rather early and yields the second maximum very close to the first one so they are almost indistinguishable from each other. However, the second one but produces a wing in the standard deviation of time dependence for larger times (Fig. 7b).

The coincidence of characteristic moments observed in time dependence of water mass, temperature, aver-

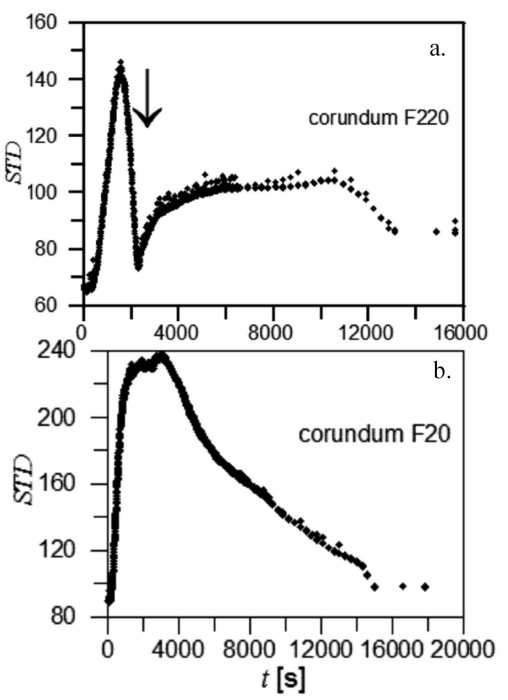

Fig. 7. Time dependence of the standard deviation of brightness of the image of (a) F220 and (b) F20 grain size samples. The arrow in (a) indicates the end of the CRP.

age brightness and STD for fine grain sample should be underlined. The linear decline in sample mass ends at $2300 \mathrm{~s}$ (Fig. 1a). As mentioned before, the time position of the first dip occurring at the end of the first step in the plot of temperature vs. time is $\approx 2300 \mathrm{~s} \mathrm{(Fig.} \mathrm{2a).}$ The change of slope of the average brightness vs. time was found also at $\approx 2300 \mathrm{~s}$ (Fig. 6a). The profound minimum found after the first peak in STD vs. time plot occurs also for $2300 \mathrm{~s}$. It means that the end of the first drying period (CRP) leaves marked signatures on time evolution of most quantities observed during drying process for the fine grain sample.

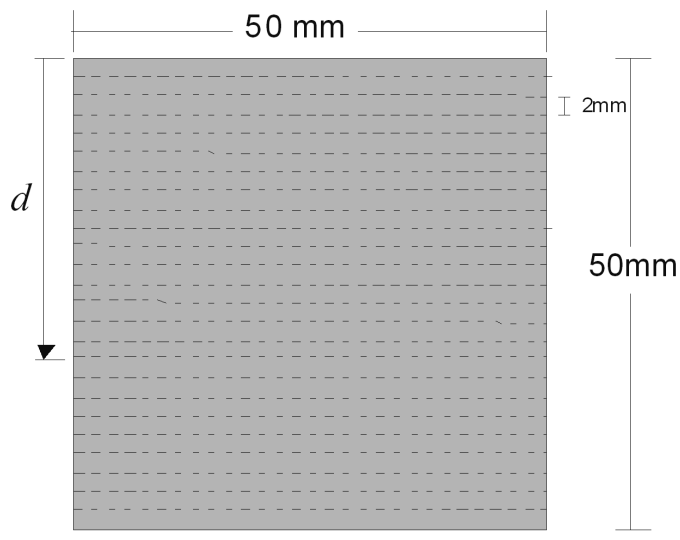

Fig. 8. Sketch of the positions of the segments for analysis of the development of statistical parameters of sample image during drying.

In order to quantify the kinetics of drying at various parts of the sample the average brightness and its standard deviation was calculated for horizontal segments 


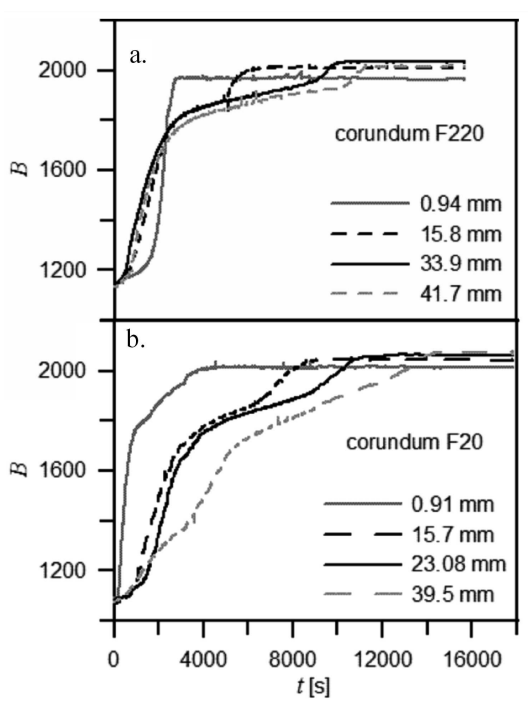

Fig. 9. Examples of time dependence of the average brightness on the indicated segments (Fig. 8) of the image of (a) F220 and (b) F20 grain size samples.
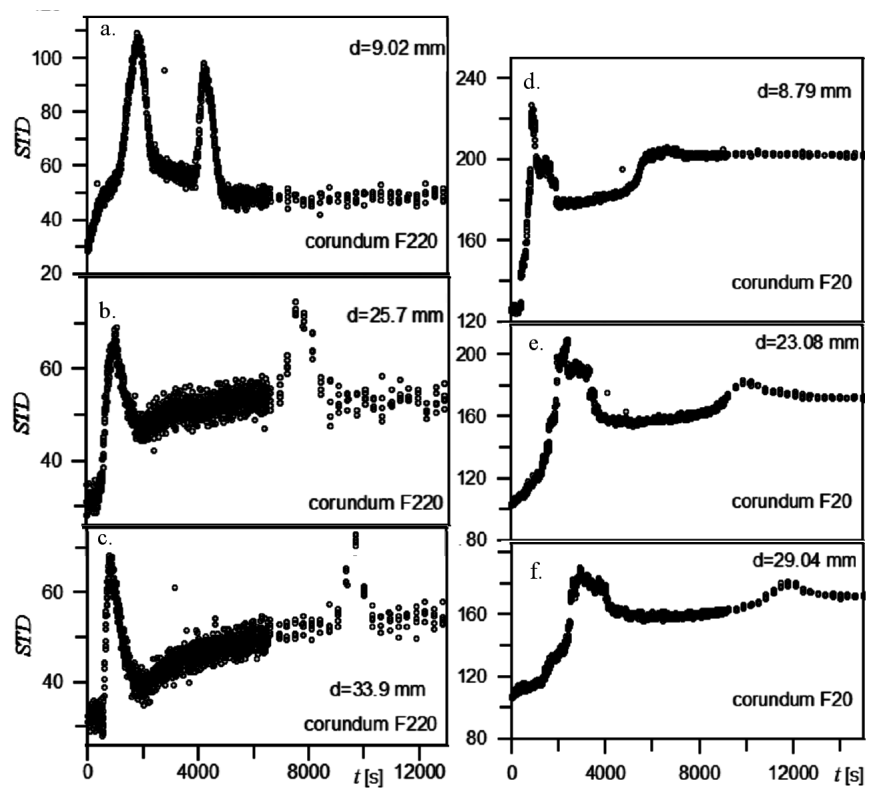

Fig. 10. Examples of time dependence of standard deviation of brightness for indicated segments (Fig. 8) in the image of F220 (a,b,c) and F20 (d,e,f) grain size samples.

at various distances $d$ from the open upper sample surface (Fig. 8). The 23 such approximately equidistant $(\approx 2 \mathrm{~mm})$ segments were analyzed for each experiment. The plots of the average brightness for each segment exhibit the initial steep increase accompanied (for segments positioned not to close to the open surface) with the discernible step-like behavior for larger times (Fig. 9). This pattern is much more visible for the fine grain sample than for the coarse grain one. The effect can be ex-

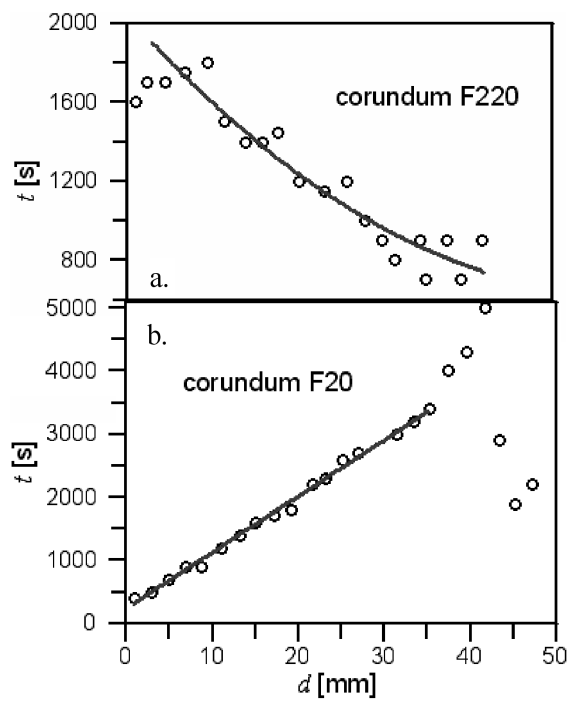

Fig. 11. The dependence of the time position of the first maximum in the standard deviation of brightness for the F220 (a) and the F20 (b) corundum sample on the depth of the segment as depicted in Fig. 8.

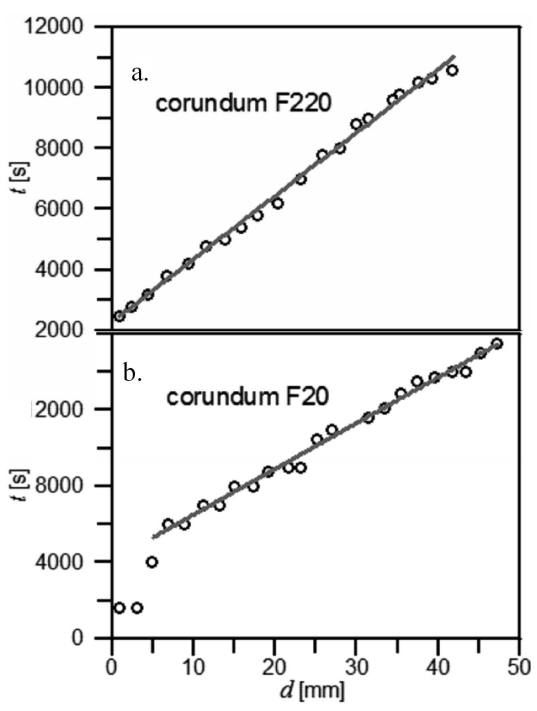

Fig. 12. The dependence of the time position of the second maximum in the standard deviation of brightness for the F220 (a) and the F20 (b) corundum sample on the depth of the segment as depicted in Fig. 8.

plained by more erratic water movement during drying of the coarse grain medium as compared to rather smooth changes in liquid spatial distribution inside the fine grain material.

The investigated time dependence of the standard deviation is very characteristic since two profound maxima are easily visible in the plots of STD vs. $t$ (Fig. 10). The feature is independent of the grain size and is similar to that one found for the standard deviation for whole sample. The time position of the first maximum increases with the segment depth for corundum of larger grains 
whereas it decreases for the small grain sample (Fig. 11). This indicates that the drying of the first one starts from the outer surface whereas it develops from the inside in case of the latter. However, the second maximum time position increases with the segment depth for both samples (Fig. 12) confirming that it is due to the drying front moving from the top surface. The average rates of motion of the front is $4.2 \mu \mathrm{m} \mathrm{s}^{-1}$ and $4.8 \mu \mathrm{m} \mathrm{s}^{-1}$ for coarse and time grain medium, respectively.

\section{Conclusions}

It was found that the commonly recognized periods of drying are not identifiable with classical mass and temperature measurements for the medium consisting of large grains. However, the statistical analysis of the sample images permitted the identification of the main periods of drying for both particulate corundum samples. The discernible periods of drying are particularly well observed for the fine grain material and marked signatures of the end of first period of drying were revealed in sample mass, temperature, average image brightness, as well as standard deviation of brightness time evolutions. It was found that in the initial phase of drying the inner parts of the sample are more affected by water evaporation than the superficial ones. Although the fully fledged chaotic and turbulent nature of drying was observed in the coarse grain medium, the similar feature was found for the fine grain material, although reduced significantly. The time dependence of the standard deviation of the im- age brightness reveals two separate maxima which help to distinguish the first of the drying period from the advanced one when the discernible drying front travels from the open surface into the body of the sample.

\section{References}

[1] J. van Brakel, in: Advances in Drying, Ed. A.S. Mujumdar, Hemisphere, New York 1980, p. 217.

[2] G.W. Scherer, J. Am. Ceram. Soc. 73, 3 (1990).

[3] E.U. Schlünder, Drying Technol. 22, 1517 (2004).

[4] A.V. Luikov, Int. J. Heat Mass Transfer 18, 1 (1975).

[5] I. Fijał-Kirejczyk, J.J. Milczarek, J. Banaszak, A. Trzciński, J. Żołądek, Nukleonika 54, 123 (2009).

[6] I.M. Fijał-Kirejczyk, J.J. Milczarek, J. ŻołądekNowak, Nucl. Instrum. Methods Phys. Res. A 651, 201 (2011).

[7] I.M. Fijał-Kirejczyk, J.J. Milczarek, J. ŻołądekNowak, F.C. de Beer, M.J. Radebe, G. Nothnagel, Acta Phys. Pol. A 122, 410 (2012).

[8] I.M. Fijał-Kirejczyk, J.J. Milczarek, F.C. de Beer, M.J. Radebe, G. Nothnagel, J. Żołądek-Nowak, Nukleonika 57, 529 (2012).

[9] I.M. Fijał-Kirejczyk, J.J. Milczarek, M.J. Radebe, F.C. de Beer, G. Nothnagel, J. Żołądek-Nowak, Drying Technol. 31, 872 (2013).

[10] N. Shokri, P. Lehmann, P. Vontobel, D. Or, Water Resour. Res. 44, W06418 (2008). 